\title{
Acute Upper Airway Obstruction Secondary to Retained Respiratory Secretions in a Patient with SARS-CoV-2 Respiratory Failure Treated with Prolonged CPAP
}

\author{
David Jones ${ }^{1}$, Veena Surendrakumar ${ }^{1}$, Ismail Vokshi ${ }^{1}$, Nicholas Oscroft ${ }^{1}$
}

\section{Abstract}

SARS-CoV-2 is a novel coronavirus which can cause respiratory failure requiring ventilatory support. We describe the first documented case of life-threatening upper airway obstruction due to retained secretions around a nasogastric (NG) tube in a patient suffering with this disease who was managed with prolonged continuous positive airways pressure (CPAP). This case highlights the need for awareness around the development of an upper airway mass due to tenacious secretions in SARS-CoV-2 positive patients and the need to develop preventative strategies in order to prevent avoidable mortality.

Keywords: Airway obstruction; CPAP; SARS-CoV-2; Coronavirus; Nasogastric tube; Non-invasive ventilation

\section{Introduction}

In March 2020 SARS-CoV-2, a singlestranded RNA virus that primarily spreads via respiratory droplets, was declared a pandemic by the World Health Organisation [1]. Presentation of SARS-CoV-2 can range from mild self-limiting viral symptoms to severe multi-organ dysfunction including respiratory failure [2], with mortality in the UK reported as $14 \%$ in those tested [3]. Critically unwell patients often require prolonged medical support as no proven treatments are available to treat the virus itself [4]. Given the advice against the use of high flow nasal oxygen in this setting [5]; for some patients, especially those deemed not appropriate for intubation, this may mean prolonged periods of non-invasive respiratory support. We present a case of a patient who received prolonged continuous positive airways pressure (CPAP) therapy for SARS-CoV-2 respiratory failure who developed acute airway obstruction due to retained secretions, requiring emergency intubation and manual removal of the obstructing mass.

\section{Case Report}

A 73-year-old fully independent female (Rockwood Frailty Score [6]:3) presented to hospital with a week history of general malaise, reduced appetite, dry cough and diarrhoea. She had a history of hypothyroidism and curative mastectomy for breast cancer five years previously. On admission, the patient was hypoxaemic on room air $\left(\mathrm{PaO}_{2} 7.66 \mathrm{kPa}\right)$, with raised inflammatory markers (white cell count $6.6 \mathrm{x}$ 109/1, C-reactive protein $230 \mathrm{mg} / \mathrm{l}$ ). Chest radiograph showed bilateral patchy peripheral and basal predominant consolidation consistent with SARS-CoV-2 pneumonitis. Prior to confirmation of SARSCoV-2, the patient was managed with supplementary oxygen, antibiotics and intermittent self-proning. However, she was unable to maintain normoxaemia despite an $\mathrm{FiO}_{2}$ 0.7, warranting urgent transfer to critical care for initiation of CPAP.

After admission to critical care the patient became dependent on CPAP, which she received using a full facemask at $10 \mathrm{~cm} \mathrm{H}_{2} \mathrm{O}$, humidified with a heat and moisture exchange (HME) filter. Given her clinical condition and in concordance with the patient's wishes, a decision was made that she would not be for intubation should her SARS-CoV-2 pneumonitis worsen and a 'Recommended Summary Plan for Emergency Care and Treatment' (ReSPECT) form [7] completed. The patient required no additional organ support; however, due to her CPAP dependence, enteral feeding was started via a nasogastric (NG) tube.

Over the next ten days, although CPAP dependent, the patient showed steady improvement, as evidenced by a reduction in her $\mathrm{FiO}_{2}$ to 0.3 and improvements in serial chest radiographs. She remained alert and orientated.

On day 18 of CPAP therapy, concerns were raised about the NG tube, which could not be flushed. A chest radiograph confirmed correct positioning, however, the clinical team were unable to withdraw the tube. Therefore, it was decided to attempt a flexible nasal endoscopy to visualise the cause of the obstruction.

Prior to the endoscopy, the patient developed sudden onset respiratory distress with oxygen

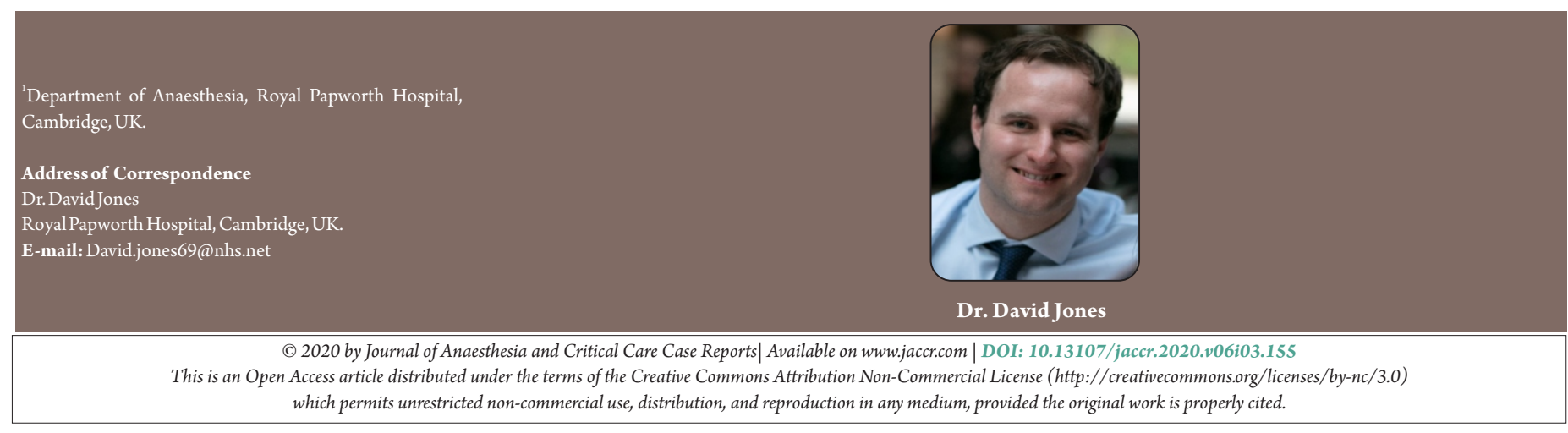

05 | Journal of Anaesthesia and Critical Care Case Reports | Volume 6 | Issue 3 | September-December 2020 | Page 05-07 
desaturation to $70 \%$. The CPAP facemask was removed immediately, and ventilation was assisted with a Mapleson C circuit. Despite optimisation of bag-mask ventilation, including the use of two-handed bagging with jaw thrust, oropharyngeal and nasopharyngeal airways, the patient continued to desaturate and began to lose consciousness. In light of the acute onset of deterioration, a decision was made to proceed with intubation.

Subsequent to induction with propofol and rocuronium, laryngoscopy with a McGrath video laryngoscope identified a significantly obscured view of the vocal cords with a large mass obstructing the laryngopharynx. A size six endotracheal tube was successfully passed over a bougie to secure the airway, and McGill's forceps used to gently remove the large mass and adherent nasogastric tube (figure 1). The hypoxia resolved following intubation.

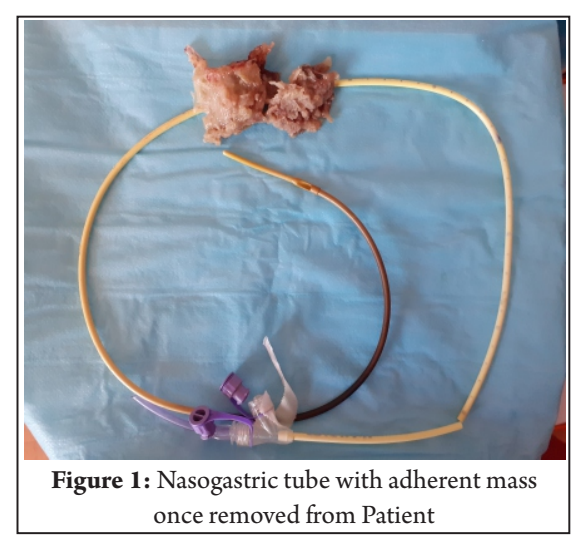

Following a period of clinical stability under sedation, a percutaneous tracheostomy was performed, and the patient was subsequently woken. No neurological injury was observed as a result of the hypoxia. Ventilatory support was weaned over the next two weeks, with successful decannulation of her tracheostomy. She was subsequently discharged to the general medical ward after 35 days in critical care.

Microbiological analysis of the obstructive mass revealed a combination of candida krusei, mixed skin flora, pus cells and epithelial cells.

\section{Discussion}

Tenacious secretions appear to form part of the clinicopathological presentation of SARS-CoV-2 and blockage of endotracheal tubes has been documented on multiple occasions [8]. However, this phenomenon has not previously been described outside of invasively ventilated patients. To our knowledge, this is the first documented case of acute upper airway obstruction related to the solidification of secretions around a NG tube in a SARS-CoV-2 positive patient treated with prolonged CPAP.

Use of CPAP may help avoid invasive mechanical ventilation in patients with respiratory distress, improving outcomes and reducing resource use [5]. However, in this case, a complication of therapy proved near fatal. We hypothesise that this event may have occurred due to a number of risk factors, including SARS-CoV-2 positive status, duration of CPAP therapy, not using an external humidification system, difficulties with mouth care and the presence of a nasogastric tube. We theorise that the mass developed progressively over time until it reached a critical size causing upper airway obstruction. Additionally, development of the mass may have been responsible for the patient's prolonged dependence on CPAP. Possible preventative strategies in this case include routine thorough mouthcare to reduce secretion burden (both in terms of volume and viscosity) in addition to the use of including mucolytic medications and chest physiotherapy. External humidification of CPAP in SARS-CoV-2 is not currently recommended due to infection control concerns [5] but could potentially also reduce the tenacity of secretions and warrants further review. Regular review of the need for nasogastric feeding or elective changing of a NG tube could also be used to reduce the possibility of a mass forming around it. Furthermore, video nasal endoscopy could be used to assess the upper airway if there is suspicion of a mass accumulating to allow planning of management strategies before the mass reaches a critical size.

The decision to intubate this patient at the time of acute deterioration raises ethical issues. The recent implementation of the 'ReSPECT' forms in the UK attempt to create personalised recommendations for patient care in possible future emergency situations [7]. These have been designed to reflect both patient preferences and the clinical judgement of the patient's medical team to avoid medical interventions that are unlikely to be successful and/or against a patient's wishes. This case highlights the importance of the context of these difficult decisions. In the scenario of progressive SARS-CoV-2 pneumonitis intubation may not have been appropriate, however acute upper airway obstruction of uncertain aetiology in an improving patient was reasonably felt to need appropriate emergency intervention, in this case leading to a positive outcome. This emphasises the importance of effective clinical judgement with consideration of both the circumstances and context especially when deciding treatment options in emergency situations. This adaptable way of decision-making is supported by the General Medical Council [9] and should be remembered when encountering difficult situations relating to life-saving treatment.

\section{Conclusion}

This case proves that the previously documented tenacious secretions associated with SARS-CoV-2 induced pneumonitis can progress to complete airway obstruction with associated mortality and morbidity in noninvasively ventilated patients. We hope by raising awareness of this phenomenon and by highlighting the associated risk factors and potential preventative strategies we reduce the future incidence of similar events occurring due to this novel disease.

Furthermore, this case demonstrates that clinical decisions should always be made in context and be adaptable, considering all of the available information even when patients wishes have been clearly documented. This is especially true with regards to limitations of treatment and resuscitation where acute deteriorations can be related to a reversible pathology that should be readily explored to prevent avoidable mortality. 


\section{References}

1. World Health Organization [Internet]. WHO Timeline - COVID-19. 27th April 2020. [Cited 22nd May 2020]. Available from https://www.who.int/news$\mathrm{room} /$ detail/27-04-2020-who-timeline---covid-19

2. Intensive care national audit \& research centre (ICNARC) [Internet]. COVID-19 Report. 15th May 2020. [Cited 22nd May 2020]. Available from https://www.icnarc.org/Our-Audit/Audits/Cmp/Reports

3. GOV.UK [Internet]. Coronavirus (COVID-19) in the UK. 21st May 2020. [Cited 22ndMay 2020].Availablefrom https://coronavirus.data.gov.uk/

4. Sanders JM, Monogue ML, Jodlowski TZ, Cutrell JB. Pharmacologic Treatments for Coronavirus Disease 2019 (COVID-19): A Review [published online ahead of print, 2020 Apr 13]. JAMA. 2020;10.1001/jama.2020.6019. doi:10.1001/jama.2020.6019

5. NHS England and NHS Improvement. Guidance for the role and use of non-invasive respiratory support in adult patients with COVID-19 (confirmed or suspected). Speciality guides for patient management during the coronavirus pandemic, 2020.
[Cited 19th June 2020] Availablefrom https://www.england.nhs.uk/coronavirus/wpcontent/uploads/sites/52/2020/03/specialty-guide-NIV-respiratory-supportand-coronavirus-v3.pdf.

6. K. Rockwood et al. A global clinical measure of fitness and frailty in elderly people. CMAJ2005; 173:489-495.

7. Resuscitation Council (UK) [Internet]. ReSPECT. c2014-2020. [cited 22nd May 2020].Availablefrom: https://www.resus.org.uk/respect/

8. Rubano JA, Jasinski PT, Rutigliano DN, et al. Tracheobronchial Slough, a Potential Pathology in Endotracheal Tube Obstruction in Patients with Coronavirus Disease 2019 (COVID-19) in the Intensive Care Setting [published online ahead of print, 2020May 20].Ann Surg. 2020;10.1097

9. General Medical Council [Internet]. Cardiopulmonary resuscitation. c2020. [cited 22nd May 2020]. Available from: https://www.gmc-uk.org/ethicalguidance/ethical-guidance-for-doctors/treatment-and-care-towards-the-end-oflife/cardiopulmonary-resuscitation-cpr
Conflict of Interest: Nil

Source of Support: None

\section{How to Cite this Article}

Jones D, Surendrakumar V, Vokshi I, Oscroft N | Acute Upper Airway Obstruction Secondary to Retained Respiratory Secretions in a Patient with SARS-CoV-2 Respiratory Failure Treated with Prolonged CPAP | Journal of Anaesthesia and Critical Care Case Reports | September-December 2020; 6(3): 05-07. 